\title{
The Observer XT: A tool for the integration and synchronization of multimodal signals
}

\author{
Patrick H. Zimmerman \\ Noldus Information Technology, Wageningen, The Netherlands \\ J. Elizabeth Bolhuis \\ Wageningen Institute of Animal Sciences, Wageningen, The Netherlands \\ AND \\ Albert Willemsen, Erik S. Meyer, and Lucas P. J. J. Noldus \\ Noldus Information Technology, Wageningen, The Netherlands
}

\begin{abstract}
The Observer was originally developed as a manual event recorder for the collection, management, analysis, and presentation of observational data in animals. Because of the flexibility of later versions, it became clear that The Observer was suitable for almost any study involving collection of observational data in both animals and humans. Furthermore, the most recent version of The Observer (The Observer XT) allows the integration and synchronization of multimodal signals from various sources, such as observational, video, tracking, and physiological data. This article describes how The Observer XT was used to integrate and synchronize video, observational, tracking, and physiological data from an experiment carried out in 2001 at the Wageningen Institute of Animal Sciences of Wageningen University and Research Centre. The integration and synchronization of these multimodal signals in The Observer XT allows the user to draw a more complete picture of the phenomena under study.
\end{abstract}

The Observer was originally developed as a manual event recorder for the collection, management, analysis, and presentation of observational data in animals (Noldus, 1991). However, because of its flexibility, it soon became clear that The Observer was suitable for almost any study involving collection of observational data. Since then, The Observer has been used in a wide range of research areas, such as zoology (e.g., Pilz, Quiroga, Schwabl, \& Adkins-Regan, 2004), entomology (e.g., Gols et al., 2005), neuroscience (e.g., Abrahams et al., 2005), psychology (e.g., Marlier \& Schaal, 2005), human-computer interaction (e.g., Kaikkonen, Kekäläinen, Cankar, Kallio, \& Kankainen, 2005), sports sciences (e.g., James, Mellalieu, \& Jones, 2005), and applied ethology (e.g., Morrison, Hemsworth, Cronin, \& Campbell, 2003).

In The Observer XT 8.0, you can import prerecorded video files and carry out detailed offline observations. Furthermore, you can carry out live observations while simultaneously creating video files from within The Observer using a USB or FireWire camera and acquiring physiological data using an external data acquisition (DAQ) device, which can be either an analog or a digital system. The external physiological data is imported into The Observer after an observation. Synchronization of the observational data and physiological data can be achieved automatically by sending out a synchronization signal during an observation from the Observer computer to the DAQ device via the COM or USB port of the Observer computer. This synchronization signal is acquired by the DAQ device and contains an almost continuous stream of time and date information. This time and date information is used, upon import of the physiological data file into The Observer XT, to synchronize the observational and physiological data. If, during live scoring, you record a video file from within The Observer, this video file is also automatically synchronized with the corresponding observation.

In animal behavior and welfare research, more and more studies combine behavioral observations with measurement of other types of data such as heart rate (e.g., Driessen, Peeters, \& Geers, 2008; Innes \& McBride, 2008; Parker et al., 2004; Schmied, Waiblinger, Scharl, Leisch, \& Boivin, 2008; Valance et al., 2008). The benefit of combining different modalities is that you get a more complete picture of the phenomena you are studying, but measuring different modalities often means that the data are acquired by different programs, thereby introducing synchronization and integration issues.

In the novel environment test, used as an example in this article, video, observational, and tracking data were initially collected using three separate programs. The

P. H. Zimmerman, patrick.zimmerman@noldus.nl 
novel environment test was carried out to examine the effects of coping style (also called personality type) and housing conditions on a pig's behavioral and physiological response to this novel environment. Coping style is the consistent behavioral and neuroendocrine response of an animal to certain challenging situations (Koolhaas et al., 1999); in pigs, the existence of diverging coping styles has been demonstrated (Bolhuis, Schouten, De Leeuw, Schrama, \& Wiegant, 2004; Bolhuis, Schouten, Schrama, $\&$ Wiegant, 2005b; Hessing, Hagelsø, Schouten, Wiepkema, \& Van Beek, 1994; Ruis et al., 2000). A pig's coping style can be determined by a backtest, in which the pig is manually restrained in a supine position during the suckling period and characterized on the basis of its behavioral response - that is, either high-resisting (HR) or low-resisting (LR) (Bolhuis, Parmentier, Schouten, Schrama, \& Wiegant, 2003; Hessing et al., 1993). Recently, it has been shown that the impact of environmental conditions on behavior differs for pigs with diverging responses in the backtest (Bolhuis, Schouten, Schrama, \& Wiegant, 2005a). Also, the housing conditions in a pig's early life may have profound effects on its behavior and welfare later on (Schouten, 1986).

This article aims to show that The Observer XT is a useful tool to integrate and synchronize data from different sources. Multimodal data was used from the novel environment test mentioned above. This test was conducted in 2001 at the Wageningen Institute of Animal Sciences. To acquire data on the behavior of the pigs, three separate programs were used for this experiment. The Observer XT enables the synchronized visualization and analysis of multimodal signals, thereby providing a more complete picture of the pigs' responses to the novel environment.

\section{METHOD}

For the novel environment test, 80 Great Yorkshire $\mathrm{x}$ (Great Yorkshire $x$ Dutch Landrace) pigs were selected on the basis of their backtest response and housed in eight groups of 10 pigs each, in either substrate-rich ("enriched," E) pens, or pens without substrate ("barren," B). When the pigs were 10 weeks of age, housing conditions were changed for half of the groups (B-E, E-B), whereas for the other groups the housing conditions remained unchanged (B-B, E-E). This was done to distinguish between the effect of actual housing conditions and housing conditions early in life on the behavioral and physiological response of the pigs in the novel environment test.

The novel environment test was carried out on each pig at the age of 16 weeks. The novel environment was an open field of approximately $5 \times 5 \mathrm{~m}$. The pig was exposed to the novel environment for a total of $10 \mathrm{~min}$. After $5 \mathrm{~min}$, a novel object (a bucket lowered from the ceiling by a rope) was introduced. The behavior and sound of the pig in the 10-min test were recorded on VHS videotape by a camera and microphone mounted on the ceiling above the open field. Before a pig entered the novel environment, it was equipped with the Polar sports watch (model S810i) to measure its heart rate; a chest strap with electrodes was attached to the animal, and the receiver-watch was fixed onto its back. Just before the animal entered the novel environment, data acquisition on the Polar system, visible in the camera image, was started. Immediately after the pig had entered the novel environment through the entrance door and the door was shut behind it, the observation of the exploratory behavior was started, using a handheld computer with The Observer 2.0 mo- bile installed during the 10-min novel environment test. Afterward, the number of vocalizations were scored from videotape with The Observer 3.0, the current version of The Observer at the time of the test. EthoVision 3.1 was used to determine the locomotory behavior and the location of the animal in the novel environment, also using the recording on videotape.

The difference in offset between heart-rate measurement and the observation on the handheld computer was determined with a stopwatch. Afterward, the heart-rate data acquired before the start of the observation were manually removed to synchronize the observational "live" data and the heart-rate data. The offline observation from videotape to determine the number of vocalizations was started at the same time as the start of the live observation, the moment the door was closed after the pig had entered the novel environment. Also, tracking in EthoVision was started at approximately the same time point in the video. All these data were entered in an Excel spreadsheet for further visualization and analysis. At the time of the novel environment test (November 2001), it was not possible to integrate and synchronize data in The Observer 3.0. Therefore, data were acquired from different programs and manually integrated and synchronized in an Excel spreadsheet. If the experiment were carried out today using The Observer XT 8.0 (see the Results section to read about the integration of the 2001 data into The Observer XT 8.0), integration and synchronization of the multimodal signals would be possible. As a result, data analysis would be more precise and faster.

\section{RESULTS}

This section describes how the multimodal signals were integrated and synchronized in The Observer XT. First, the recording of the novel environment test of a pig was digitized from VHS videotape to an MPEG-2 video file using the Noldus MPEG recorder. The two observational data files (*.odf) from The Observer 3.0 (one with behaviors, the other one with vocalizations and the appearance of the novel-object-as-a-point event) were imported into The Observer XT, resulting in two separate observations. The associated digital video file was imported into both observations. The first occurrence of snout floor and novel object was used to synchronize the video file and the observational data file with behaviors and vocalizations, respectively. Next, the Polar heart-rate file was imported into The Observer XT as an external data file. The start of the heart-rate file was synchronized with the video, using the visible start of data acquisition before the test. The locomotory behavior of the pig was analyzed in EthoVision XT 5.0; the video file was opened, and tracking started at the moment the pig entered the open field. In EthoVision XT, two zones, "edge" and "center," were created to track the location of the pig in the open field. The tracking data were exported and the Noldus Zone transitions macro was used to create a file with State events "edge" and "center." This EthoVision XT export file was imported into The Observer XT as an observational data file. The EthoVision XT export file was synchronous with the start of the video file.

The result of the integration and synchronization in The Observer XT is shown in Figure 1.

The integration and synchronization of observational, tracking, video, and physiological data allows one to determine, for example, whether animals with diverging coping styles behave differently in the novel environment 


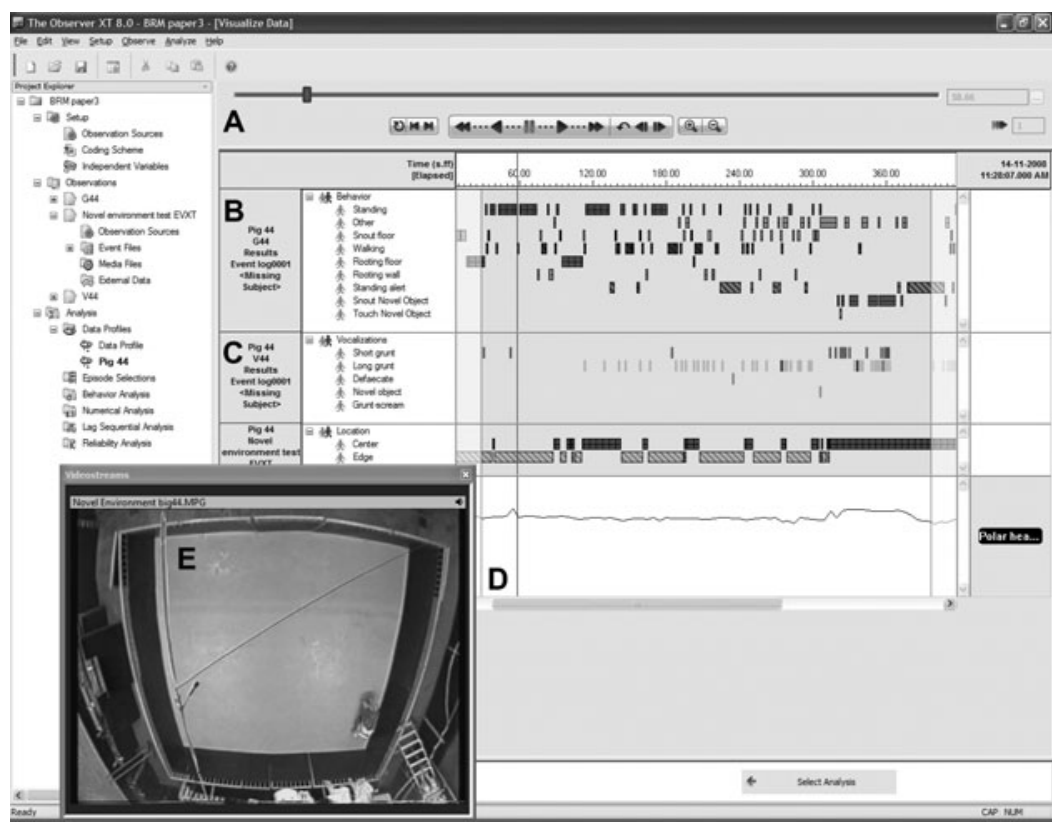

Figure 1. Screenshot of the visualization of all signals in The Observer XT. (A) The playback control window that allows synchronous playback of the data. (B) The timeevent plot of the behavioral data. (C) The time-event plot of the vocalizations. (D) The graph of the heart-rate data. (E) The video file of the pig in the open field. The vertical line in the time-event plots and heart-rate graph represents the position in the observation that corresponds to the current frame in the video file.

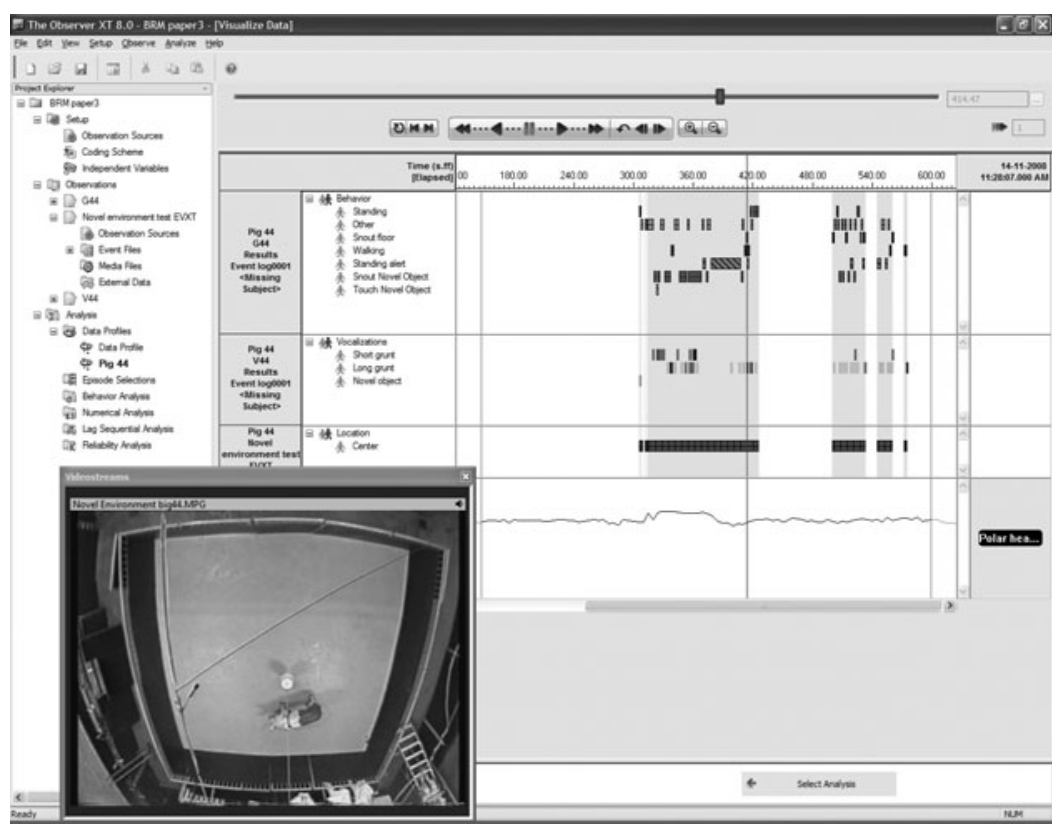

Figure 2. The visualization of data selection in The Observer XT. The intervals depicted in the time-event plots are the ones in which the pig was in the "center" of the open field. Also, data is selected only for the period after the novel object appeared; all other data are excluded. 


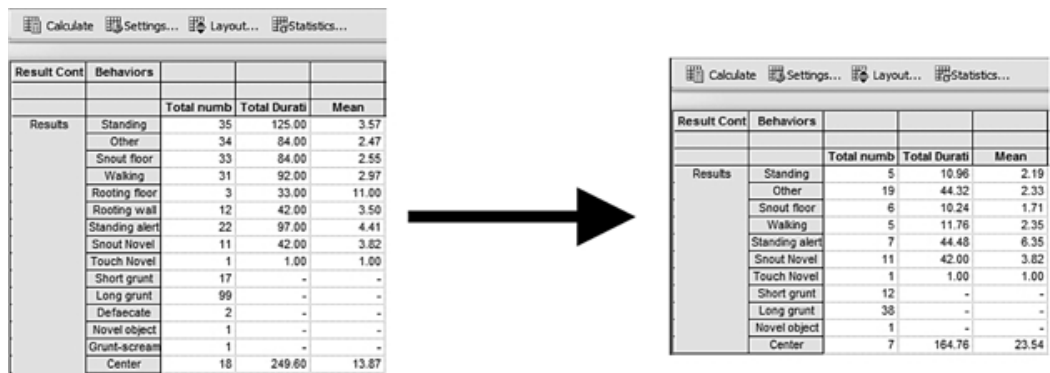

Figure 3. The result of data selection in the behavioral analysis results sheet: The sheet on the left shows all data; the sheet on the right shows the statistics for the behaviors and vocalizations when the pig was in the center of the open field, after the novel object had appeared.

test. Furthermore, the integration facilitates the investigation of the relation between location and behavior. In Figure 2, you see the result of data selection in The Observer: Only location "center" has been selected, and a time interval "from appearance of novel object until the end of the observation" has been selected.

The data selection shown in Figure 2 can also be used for analysis in The Observer XT. Figure 3 shows two analysis results sheets; the one on the left shows all the data, the one on the right shows the data based on data selection, as described for Figure 2.

\section{CONCLUSION}

When measuring multimodal signals, the integration and synchronization of data, usually measured with different programs, can be a challenge, but The Observer XT can help. Automatic synchronization of observational and physiological data acquired with an external DAQ system can be achieved by sending out a synchronization signal through a cable that connects the computer running The Observer and the DAQ device. When the computer running The Observer and the DAQ device cannot be physically connected, synchronization can be achieved manually by, for example, creating a cue in the physiological signal that is simultaneously scored in The Observer XT. Physiological data in ASCII format and sampled with a constant sample rate can be imported into The Observer XT. In The Observer, you can then visualize the physiological data, but it is also possible to export part of your observational data with the corresponding physiological data. For example, you can export the heart-rate data for the period when the pig was in the center of the open field, after the novel object had appeared. These physiological data can then be imported into a spreadsheet program so that further calculations can be carried out.

Synchronization of observational and video data is achieved by creating a video file within The Observer using a USB or FireWire camera. Alternatively, when a video file is created using an external device that is not connected to The Observer, you can use a visual or auditory cue in the video image that is simultaneously scored "live" in The Observer, thereby ensuring synchronization of observational and video data.

Synchronization of observational data and tracking data (from EthoVision XT) is achieved by using the same video file. By using a Zone transitions macro, the In-Zone dependent variables and other behavioral states can be imported into The Observer XT as observational data. This allows you, for example, to investigate the behavior of the animals in relation to their location, or to relate the activity as recorded by EthoVision to specific exploratory behaviors as scored in The Observer XT.

In conclusion, The Observer XT is a useful tool to integrate and synchronize multimodal signals. This allows you to investigate relationships between different signals and, as a result, to get a more complete picture of the behavior of your subjects, thereby enhancing the quality of your research.

\section{AUTHOR NOTE}

The authors affiliated with Noldus Information Technology have a direct financial interest in The Observer and some other software described in this article. Correspondence concerning this article should be addressed to P. H. Zimmerman, Noldus Information Technology, P.O. Box 268, $6700 \mathrm{AG}$, Wageningen, The Netherlands (e-mail: patrick .zimmerman@noldus.nl).

\section{REFERENCES}

Abrahams, B. S., Kwok, M. C. H., Trinh, E., Budaghzadeh, S., HosSAIN, S. M., \& Simpson, E. M. (2005). Pathological aggression in "fierce" mice corrected by human nuclear receptor 2E1. Journal of Neuroscience, 25, 6263-6270.

Bolhuis, J. E., Parmentier, H. K., Schouten, W. G. P., Schrama, J. W., \& Wiegant, V. M. (2003). Effects of housing and individual coping characteristics on immune responses of pigs. Physiology \& Behavior, 79, 289-296.

Bolhuis, J. E., Schouten, W. G. P., De Leeuw, J. A., Schrama, J. W., \& WiEgant, V. M. (2004). Individual coping characteristics, rearing conditions and behavioural flexibility in pigs. Behavioural Brain Research, 152, 351-360.

Bolhuis, J. E., Schouten, W. G. P., Schrama, J. W., \& Wiegant, V. M. (2005a). Behavioural development of pigs with different coping characteristics in barren and substrate-enriched housing conditions. Applied Animal Behaviour Science, 93, 213-228.

Bolhuis, J. E., Schouten, W. G. P., Schrama, J. W., \& Wiegant, V. M. (2005b). Individual coping characteristics, aggressiveness and fighting strategies in pigs. Animal Behaviour, 69, 1085-1091. 
Driessen, B., Peeters, E., \& Geers, R. (2008). Influence of olfactory substances on the heart rate and lying behaviour of pigs during transport simulation. Animal Welfare, 17, 155-160.

Gols, R., Bukovinszky, T., Hemerik, L., Harvey, J. A., Van LenTEREN, J. C., \& VeT, L. E. M. (2005). Reduced foraging efficiency of a parasitoid under habitat complexity: Implications for population stability and species coexistence. Journal of Animal Ecology, 74, 1059-1068.

Hessing, M. J. C., Hagels $\varnothing$, A. M., Schouten, W. G. P., Wiepkema, P. R., \& VAN BEEK, J. A. M. (1994). Individual behavioral and physiological strategies in pigs. Physiology \& Behavior, 55, 39-46.

Hessing, M. J. C., Hagels $\varnothing$, A. M., Van Beek, J. A. M., WiepKema, P. R., Schouten, W. G. P., \& Krukow, R. (1993). Individual behavioural characteristics in pigs. Applied Animal Behaviour Science, 37, 285-295.

INNES, L., \& McBRIDE, S. (2008). Negative versus positive reinforcement: An evaluation of training strategies for rehabilitated horses. Applied Animal Behaviour Science, 112, 357-368.

James, N., Mellalieu, S. D., \& Jones, N. M. P. (2005). The development of position-specific performance indicators in professional rugby union. Journal of Sports Sciences, 23, 63-72.

Kaikkonen, A., Kekäläinen, A., Cankar, M., Kallio, T., \& KANKAINEN, A. (2005). Usability testing of mobile applications: A comparison between laboratory and field testing. Journal of Usability Studies, 1, 4-16.

Koolhaas, J. M., Korte, S. M., De Boer, S. F., Van der Vegt, B. J., Van Reenen, C. G., Hopster, H., et AL. (1999). Coping styles in animals: Current status in behavior and stress-physiology. Neuroscience \& Biobehavioral Reviews, 23, 925-935.

Marlier, L., \& SchaAl, B. (2005). Human newborns prefer human milk: Conspecific milk odor is attractive without postnatal exposure. Child Development, 76, 155-168.
Morrison, R. S., Hemsworth, P. H., Cronin, G. M., \& Campbell, R. G. (2003). The social and feeding behaviour of growing pigs in deep-litter, large group housing systems. Applied Animal Behaviour Science, 82, 173-188.

Noldus, L. P. J. J. (1991). The Observer: A software system for collection and analysis of observational data. Behavior Research Methods, Instruments, \& Computers, 23, 415-429.

Parker, R., Watson, R., Wells, E., Brown, S., Nicol, Q., \& KNOWLES, T. (2004). The effect of blindfolding horses on heart rate and behaviour during handling and loading onto transport vehicles. Animal Welfare, 13, 433-437.

Pilz, K. M., Quiroga, M., Schwabl, H., \& Adkins-Regan, E. (2004) European starling chicks benefit from high yolk testosterone levels during a drought year. Hormones \& Behavior, 46, 179-192.

Ruis, M. A. W., Te Brake, J. H. A., Van de Burgwal, J. A., De Jong, I. C., Blokhuis, H. J., \& KoolhaAs, J. M. (2000). Personalities in female domesticated pigs: Behavioral and physiological indications. Applied Animal Behaviour Science, 66, 31-47.

Schmied, C., Waiblinger, S., Scharl, T., Leisch, F., \& Boivin, X. (2008). Stroking of different body regions by a human: Effects on behaviour and heart rate of dairy cows. Applied Animal Behaviour Science, 109, 25-38.

Schouten, W. G. P. (1986). Rearing conditions and behavior in pigs. Unpublished doctoral dissertation, Wageningen Agricultural University, The Netherlands.

Valance, D., Boissy, A., Després, G., Arnould, C., Galand, C., Favreau, A., ET AL. (2008). Changes in social environment induce higher emotional disturbances than changes in physical environment in quail. Applied Animal Behaviour Science, 112, 307-320.

(Manuscript received November 17, 2008; revision accepted for publication December 2, 2008.) 